\title{
Prostate volume predicts high grade prostate cancer both in digital rectal examination negative (ct1c) and positive (zct2) patients
}

\author{
Hasan Yilmaz', Murat Ustuner ${ }^{1}$, Seyfettin Ciftci ${ }^{1}$, Ufuk Yavuz¹, Tayyar Alp Ozkan², Ozdal Dillioglugil' \\ ${ }^{1}$ University of Kocaeli, School of Medicine, Urology Department, Kocaeli, Turkey; ${ }^{2}$ Derince Training and \\ Research Hospital, Urology Department, Kocaeli, Turkey
}

\section{ABSTRACT}

Introduction: We aimed to assess the relationship between prostate volume (PV) and high grade prostate carcinoma (HGPCa) in patients with benign and suspicious digital rectal examination (DRE) in our prostate biopsy cohort.

Materials and methods: Between 2009-2012, 759 consecutive initial transrectal systematic 12 cores prostate biopsies were included. PVs were calculated with transrectal ultrasound. Only prostate adenocarcinomas ( $\mathrm{PCa}$ ) were included into the study. For standardization, patients with missing data, and who have been exposed to any form of hormonal or radiation therapy were excluded. Patients were categorized with DRE (negative or positive) and Gleason sum [<7: low grade PCa(LGPCa), $\geq 7$ : HGPCa].

Results: Median PV was significantly lower in patients with HGPCa. There was a significantly increased risk of HGPCa with PV according to all groups in univariate logistic regression (LR). The significant relationship continued in multivariate LR with PSA and age. From the ROC curve analyses, again a significantly statistical concordance was found between the detection of HGPCa and PV (AUC:0.63, p<0.001), as well as between HGPCa and tPSA (AUC:0.73, $\mathrm{p}<0.001)$. tPSA and PV were also significantly concordant with HGPCa both in DRE negative and positive patients.

Conclusions: There is a significant relationship between HGPCa and decreasing PV. The continued significant relationship both in DRE negative and positive patients reinforces this relation.

\section{ARTICLE INFO}

\section{Key words:}

Prostate; Prostatic Neoplasms; Digital Rectal Examination

Int Braz J Urol. 2014; 40: 613-9

Submitted for publication:

March 05, 2014

Accepted after revision:

June 23, 2014

\section{INTRODUCTION}

Prostate volume (PV) is still a hot topic in the studies for prostate cancer (PCa). Recently the importance of PV was evaluated for risk calculators of Prostate Cancer Prevention Trial (PCPT) and European Randomised Study of Screening for Prostate Cancer $(1,2)$. The relationship between smaller prostate volume (PV) and high grade prostate carcinoma (HGPCa) has been an interesting issue after the publication of PCPT (3). The inverse relationship between PV and HGPCa has been first shown by Kulkarni et al. in their prostate biopsy cohort (4). Thereafter, this inverse relationship was supported with radical prostatectomy (RP) cohorts in subsequent studies (5-7).

Recently, two different studies were submitted by the same institute for the assessment of the relationship between PV and HGPCa $(5,8)$. They initially showed in their RP cohort that there was a significant relationship between PV and HGPCa in patients with clinical stage T1c prostate cancer but not in $\geq \mathrm{T} 2$ (5). Secondly, they supported identical findings (significant relationship in 
$\mathrm{T} 1 \mathrm{c}$, but not in $\geq \mathrm{T} 2$ ) in their prostate biopsy cohort (8). We aimed to assess the relationship between PV and HGPCa in patients with benign and suspicious digital rectal examination (DRE) in our prostate biopsy cohort.

\section{MATERIALS AND METHODS}

Between 2009-2012, 1381 consecutive initial (repeat biopsies were not included) transrectal ultrasound (TRUS) guided systematic 12 cores prostate biopsies (12 $\mathrm{Bx}$ ) were evaluated retrospectively. Age, total PSA (tPSA) before biopsy, DRE, TRUS calculated PVs with the ellipse method (length $\mathrm{X}$ depth $\mathrm{X}$ width $\mathrm{X} \pi / 6$ ) were noted. Benign pathologies were categorized as non-cancer (benign) and prostate adenocarcinomas were categorized as cancer (malignant). Patients with high grade prostatic intraepithelial neoplasia and atypical small acinary proliferations were excluded for the sake of clarity of the results. For standardization, patients with missing data, cancer diagnosis other than prostate adenocarcinoma, total number of cores less or more than 12, and patients who had previous anti-androgen, 5-alfa reductase inhibitory treatment or prostatic radiation therapy were also excluded. The remaining 759 patients that met the inclusion criteria were included to study.

Standard $12 \mathrm{Bx}$ (both lateral and medial biopsies from the base, medial and apex on the right and left side of the prostatic peripheral zone) was performed for all prostate volumes, ages or tPSA levels. No transitional zone or finger guided biopsies were performed. DREs were only performed by a skilled urologist or by a last year urology resident.

Patients were categorized with DRE (benign or negative versus suspicious or positive) and Gleason sum $[<7$ : low grade PCa(LGPCa), $\geq 7$ : HGPCa]. While statistical analyses were performed, PV variable was recoded as PV/10 that was an ordinal variable and it corresponded to each 10 cc decrease of PV variable. All biopsies were evaluated by the same pathologist (BM) based on the 2005 International Society of Urological Pathology Consensus Conference on Gleason Grading of Prostatic Carcinoma (9).

\section{Statistical analysis}

DRE and Gleason sum categories were modeled as a dichotomous variable (yes/no). All data was analysed with Statistical Package for Social Science database program. The Independent Sample t test was used for continuous variables when variables were normally distributed and equal variations were assumed. The Mann-Whitney U test was used for categoric variables or for continuous variables when variables were not distributed normally or equal variations were not assumed. Chi-square test was used to find the relationship between two independent categoric variables. Univariate and multivariate logistic regression analyses were performed.

By using the categoric data for the presence of HGPCa (yes or no), the PVs were separated according to their true positive and false positive rates. The true positive rates (sensitivity) and the false positive rates (1-specificity) were used to plot a receiver operating characteristic (ROC) curve. ROC curve analyses were performed with the MedCalc v12.7.8 trial version. The area under the ROC curve (AUC) summarized discriminative ability, with pairwise testing by the DeLong test.

\section{RESULTS}

Prostate biopsy was performed in 1381 patients. Four hundered fourteen patients with incomplete data, 71 patients with HGPIN and ASAP, 5 patients with cancer diagnosis other than prostate adenocarcinoma, 53 patients with total number of cores less or more than 12 cores biopsy and 79 patients with previous prostate therapy were excluded from analysis. Thus, data on 759 patients were evaluated.

Median patient age, tPSA, and PV were 65 years (Inter quartile range (IQR) 60-72), $8.1 \mathrm{ng} / \mathrm{ml}$ (IQR 5.45-13.90) and 45.6 cc (IQR 32.3-71.20), respectively. DRE was negative in 65.6\% (498/759) of the patients. PCa, LGPCa and HGPCa were present in 37.9\% (288/759), 7.4\% (56/759), and 30.6\% (232/759) of the whole cohort, in 58.2\% (152/261), $6.9 \%(18 / 261)$ and $51.3 \%(134 / 261)$ of the DRE positive patients, and in 27.3\% (136/498), 7.6\% (38/498) and 19.7\% (98/498) of the DRE negative patients, 
respectively. As stated above, HGPCa was found significantly higher than LGPCa both in DRE positive $(p<0.0001)$ and DRE negative $(p<0.001)$ patients.

Median PV was significantly lower in patients with HGPCa in all DRE status compared to patients without HGPCa (Table-1). Additionally, tPSA was significantly higher in HGPCa patients (Table-1).

In order to evaluate whether detection of HGPCa was affected by PV, a logistic regression analysis was performed. Except for age in DRE positive patients, there was a significantly increased risk of HGPCa with each 10 cc decrease of PV (PV/10), age and logPSA in DRE negative and DRE positive patients, and in the whole cohort in univariate logistic regression (LR). In multivariate LR analysis, the significant relationship between HGPCa and PV/10 continued in the whole group, and in DRE positive and DRE negative groups, although the most powerful significant relationship was between HGPCa and logPSA. There was no significant relationship between LGPCa and PV/10 (Table-2).
In addition to analyzing the data, a ROC curve was constructed for TPSA and PV variables for the detection of PCa. From the ROC curve a significantly statistical concordance was found between the detection of HGPCa and PV (AUC:0.63, $\mathrm{p}<0.001$ ), as well as between HGPCa and tPSA (AUC:0.73, p<0.001). However, the concordance of tPSA was significantly higher compared to PV in ROC curve analyses $(p=0.002)$ (Figure-1A). tPSA and PV were also significantly concordant with HGPCa both in DRE negative $(\mathrm{p}<0.001$ and $\mathrm{p}=0.001$, respectively) and positive $(\mathrm{p}<0.001$ and $\mathrm{p}<0.001$, respectively) patients (Figures $1 \mathrm{~B}$ and 1C). But there was no significant difference between TPSA and PV for HGPCa in DRE negative patients $(p=0.262)$ (Figure 1C).

\section{DISCUSSION}

Basicly, the relationship between HGPCa and PV was assessed with two different ways in the literature: by the results of prostate biopsy or the pathology of RP. Freedland et al established a powerful relationship between smaller prostate and HGPCa in

Table 1 - Patient characteristics according to presence or absence of HGPCa.

\begin{tabular}{|c|c|c|c|c|}
\hline & & \multicolumn{2}{|c|}{ HGPCa } & \multirow{2}{*}{$\mathrm{P}$} \\
\hline & & yes & no & \\
\hline \multirow{2}{*}{ Number of patients ( } & DRE Negative & 98 & 400 & $<0.001$ \\
\hline & DRE Positive & 134 & 127 & 0.665 \\
\hline \multirow[b]{2}{*}{ Median age (IQR) ye } & DRE Negative & $65.50(60.75-71.00)$ & $64.00(57.00-69.00)$ & 0.018 \\
\hline & DRE Positive & $68.00(62.00-77.00)$ & $69.00(62.00-74.00)$ & $<0.001$ \\
\hline \multirow{3}{*}{ Median tPSA (IQR) } & DRE Negative & $9.56(6.16-13.85)$ & $6.67(5.04-9.83)$ & $<0.001$ \\
\hline & & & & \\
\hline & DRE Positive & $20.10(10.18-59.23)$ & $8.62(4.85-14.40)$ & $<0.001$ \\
\hline \multirow{3}{*}{ Median PV (IQR) cc } & DRE Negative & $41.85(31.27-63.45)$ & $49.90(36.65-75.75)$ & 0.002 \\
\hline & & & & \\
\hline & DRE Positive & $36.45(26.47-53.37)$ & $45.40(32.0-74.20)$ & $<0.001$ \\
\hline
\end{tabular}


Table 2 - Logistic regression analyses of LGPCa and HGPCa in DRE negative and positive patients.

\begin{tabular}{|c|c|c|c|c|c|c|c|c|}
\hline & & & \multicolumn{2}{|c|}{ DRE Negative } & \multicolumn{2}{|c|}{ DRE Positive } & \multicolumn{2}{|l|}{ Total } \\
\hline & & & OR $(95 \% \mathrm{Cl})$ & $P$ & OR $(95 \% \mathrm{Cl})$ & $P$ & $\mathrm{OR}(95 \% \mathrm{Cl})$ & $p$ \\
\hline \multirow[t]{3}{*}{ LGPCa } & Univariate & $\mathrm{PV} / 10$ & $1.11(0.97-1.26)$ & 0.110 & $1.01(0.85-1.19)$ & 0.909 & $1.07(0.97-1.18)$ & 0.168 \\
\hline & & Age & $1.00(0.96-1.04)$ & 0.720 & $0.97(0.92-1.02)$ & 0.326 & $0.99(0.96-1.02)$ & 0.710 \\
\hline & & $\log P S A^{*}$ & $0.31(0.12-0.80)$ & 0.015 & $0.20(0.07-0.58)$ & 0.003 & $0.26(0.13-0.52)$ & $<0.001$ \\
\hline \multirow[t]{6}{*}{$\mathrm{HGPCa}$} & Univariate & $\mathrm{PV} / 10$ & $1.12(1.03-1.22)$ & 0.008 & $1.18(1.07-1.29)$ & $<0.0001$ & $1.16(1.09-1.24)$ & $<0.001$ \\
\hline & & Age & $1.03(1.00-1.06)$ & 0.022 & $1.02(0.99-1.05)$ & 0.071 & $1.04(1.02-1.06)$ & $<0.001$ \\
\hline & & $\log P S A$ & $4.83(2.49-9.39)$ & $<0.001$ & $6.30(3.42-11.57)$ & $<0.001$ & $7.94(5.10-12.36)$ & $<0.001$ \\
\hline & Multivariate & $\mathrm{PV} / 10$ & $1.22(1.10-1.34)$ & $<0.001$ & $1.33(1.18-1.49)$ & $<0.001$ & $1.30(1.20-1.40)$ & $<0.001$ \\
\hline & & Age & $1.03(1.00-1.06)$ & 0.041 & - & - & $1.03(1.01-1.06)$ & 0.010 \\
\hline & & $\log P S A$ & $5.99(2.91-12.3)$ & $<0.001$ & $\begin{array}{c}10.02(5.06- \\
19.87)\end{array}$ & $<0.001$ & $9.62(5.91-15.66)$ & $<0.001$ \\
\hline
\end{tabular}

${ }^{*} \log P S A$, the logarythm of tPSA

Figure 1 - ROC curves. True positive and false positive rates of PV and tPSA values according to presence of HGPCa. A) The comparison between PV and tPSA in all patients. B) In DRE positive patients. C) In DRE negative patients.

A

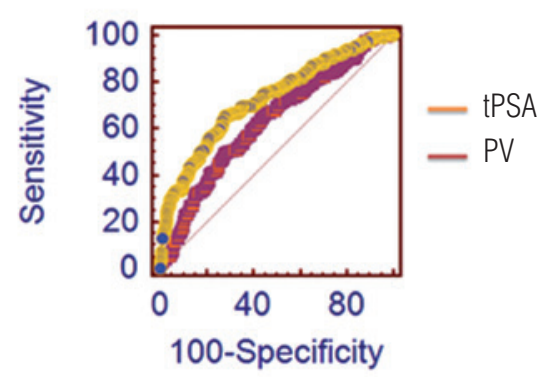

C
B

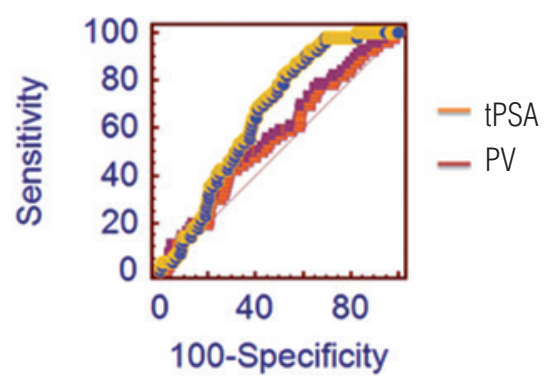

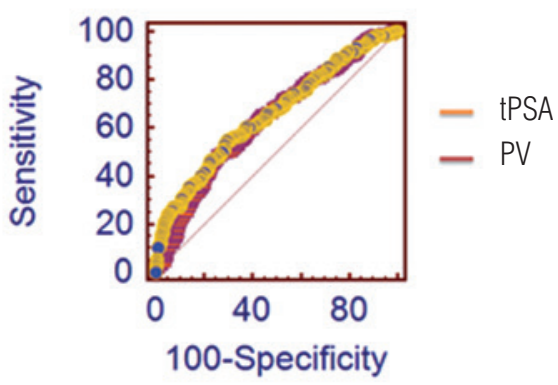


univariate and multivariate LR analyses when they rewieved their 1602 RP patients (10). Similar significant relationship was shown by Briganti et al's 4277 RP patients (6) and Newton et al's 3087 RP patients (7). Additionally, Briganti et al suggested that the relationship was pronounced at prostate volumes less than the cut-off value of $45 \mathrm{cc}$. On the other hand, Newton et al suggested that each 2 cc increase in PV, decreases the risk of HGPCa six times $(6,7)$. Kulkarni et al also found inverse significant relationship between HGPCa and PV in 369 patients with $\mathrm{TPSA}<10 \mathrm{ng} / \mathrm{ml}$ only in prostate biopsy patologies, this relationship did not continue when they reviewed these patients' RP specimens (4). However, we think that there was an important bias in their assessment of the relationship in their RP cohort. Because all of these patients had PCa at a stage that fits to a definitive therapy or their data set did not include any benign patient. Kassouf et al analyzed prostate biopsies of 247 patients (complete data missing on 3 patients) in their RP cohort (11). When they analyzed these biopsies, they found that the percentage of HGPCa below their cut-off value of 50 cc PV was 39.7\% (97/244) (11). However, they found HGPCa in 9.8\% (24/244) at PV above 50 cc. Probably because of their RP cohort, they had only 53 patients with PV above $50 \mathrm{cc}$, although 191 patients were below 50 cc (11). It seems that patients were not distributed homogeneously in Kassouf et al. study. Borden et al. found a significant relationship between HGP$\mathrm{Ca}$ and $\mathrm{PV}$ in their prospective prostate biopsy cohort although the relationship between DRE and HGPCa was their primary goal (12).

Also, the relationship between PV and HGPCa was shown in two different studies from the same institute $(5,8)$. Initially, Liu et al evaluated their 1404 RP patients (5). They claimed that the significant relationship between PV and HGPCa was shown only in clinical stage T1c patients while there was no significant relationship in the other clinical $\mathrm{T}$ stages. They concluded that the significant relation in T1c patients probably was a result of an ascertainment bias related to the performance characteristics of PSA (5). Because they believed that RP series resulted in a selection bias, Ngo et al thereafter performed an another study in a prostate biopsy cohort, and evaluated the rela- tionship between PV and HGPCa in patients with positive or negative DRE (8). They found a significant relationship in PV and HGPCa in the entire group and DRE negative patients while they did not find in DRE positive patients. They concluded that if there would be a real significant relationship, it should be shown in all groups and stages (8). In our study, we similarly evaluated our prostate biopsy cohort and found significant relationship between decreasing PV and HGPCa in DRE negative and positive patients, and in the entire group. If we follow their conclusion, there would be a real relationship between PV and HGPCa in our study because the relation continued in all groups. Despite the claims of Ngo et al, these findings reinforce the relationship between decreasing $\mathrm{PV}$ and $\mathrm{HGPCa}$.

We used also a ROC curve for the relationship between PV and HGPCa. We added the tPSA into analyses for clarifying the results. Both tPSA and PV were significantly concordant with HGPCa in total, DRE positive and negative patients according to ROC curve analyses. Not surprisingly, tPSA was significantly superior compared to PV in total and DRE positive patients. Thompson et al showed that the sensitivity and specificity of tPSA increased with high grade disease (13). They found the AUC of tPSA for all PCa was 0.68, for Gleason 7 and higher was 0.78, and for Gleason 8 and higher was 0.83. Also Optenberg et al found the AUC of tPSA for all PCa was 0.78 on the data collected between 1991-1995 years (14). However, the concordance of tPSA decreased conspicuously in DRE negative patients whereas PV was just about similar. Also there was no significant difference between PPSA and PV in DRE negative patients. This finding promotes the usefulness of PV in DRE negative patients.

In our study, we found that HGPCa was seen rarely in larger prostates (in other words prostates that have more transitional tissue ) compared to small ones. There are some hypoteses to explain this relation. First, various authors stated that this was a result of sampling error of prostate biopsy. If one claimed that there was a sampling error, this would be corrected by taking more cores. Ankerst et al evaluated the PCPT trial in terms of PV and the number of biopsy cores 6 to 10-12 
(1). They suggested that although more HGPCa was detected at lower PV, increasing core number from 6 to 10-12 was not associated with an increase in HGPCa. Elliott et al evaluated their patients $(90 \%$ of all with more than 10 cores) and anyone did not use 5- alfa reductase inhibitors (15). They found that HGPCa and any prostate cancers were detected significantly higher as PV decreased. In contrast, HGPCa was significantly predominant in below 30 cc PV (LGPCa 19\%, HGPCa 40\%), whereas LGPCa was predominant (17\% vs 14\%) above $50 \mathrm{cc}$ and they showed that the frequency of HGPCa decreased dramatically as PV increased while this was not seen with LGPCa. However, the reverse relation with HGPCa and PV was reported in Kassouf et al study ( at least 10 cores biopsy) (11) and our present study (standard 12 cores biopsy). Second, PSA increases to high levels in patients who have excessive adenoma tissue, therefore PCa is diagnosed at the early stage of the disease. However, Roehrborn et al reported that the chances in PCa detection on PSA-driven biopsies were numerically higher in the dutasteride arms; though PSA levels (28-29\%) were lower than in the tamsulosin arm (24\%) (16). On the other hand, development of HGPCa over the progression of preexisting LGPCa or de novo development is not clear. Although this obscurity, Epstein et al concluded that the tumor grade did not evolve in time (17). Thirdly, various undefined factors that grows the adenoma tissue may prevent the development of HGPCa or patients with benign prostatic hyperplasia may have genetic factors which protect against HGPCa. Further investigations may provide a better understanding to explain these hypotheses.

In contrast, the PV calculated by transrectal ultrasound generally never reflects the true PV. Definitive PV values can only be calculated with RP specimens though this leads to a selection bias. In our study, we think that we eliminated a selection bias in our prostate biopsy cohort while our PV measurements may not be the definitive values and also a Gleason upgrade can be expected at RP pathology in some patients.

\section{CONCLUSIONS}

There is a significant relationship between HGPCa and decreasing PV/10 in DRE negative and positive patients free from the limitation of selection bias originating from RP cohort. The continued significant relationship both in DRE negative and positive groups decreased the probability of the ascertaintment bias. These findings reinforce the evidence for the negative relation between HGPCa and decreasing PV.

\section{ABBREVIATIONS}

AUC $=$ Area under the ROC curve

$\mathrm{Bx}=$ Prostate biopsy

DRE = Digital rectal examination

HGPCa = High grade prostate carcinoma

IQR = Inter quartile range

LGPCa $=$ Low grade prostate carcinoma

$\mathrm{LR}=$ Logistic regression

$\mathrm{PCa}=$ Prostate adenocarcinoma

PCPT $=$ Prostate Cancer Prevention Trial

PSA $=$ Prostate spesific antigen

$\mathrm{PV}=$ Prostate volume

$\mathrm{PV} / 10$ = Each 10 cc decrease of prostate volume

ROCC $=$ Receiver operating charateristic

$\mathrm{RP}=$ Radical prostatectomy

t PSA $=$ Total prostate spesific antigen

TRUS $=$ Transrectal ultrasound

\section{REFERENCES}

1. Ankerst DP, Till C, Boeck A, Goodman P, Tangen CM, Feng Z, et al.: The impact of prostate volume, number of biopsy cores and American Urological Association symptom score on the sensitivity of cancer detection using the Prostate Cancer Prevention Trial risk calculator. J Urol. 2013;190:70-6.

2. Roobol MJ, Schröder FH, Hugosson J, Jones JS, Kattan MW, Klein EA, et al.: Importance of prostate volume in the European Randomised Study of Screening for Prostate Cancer (ERSPC) risk calculators: results from the prostate biopsy collaborative group. World J Urol. 2012;30:149-55.

3. Thompson IM, Goodman PJ, Tangen CM, Lucia MS, Miller GJ, Ford LG, et al.: The influence of finasteride on the development of prostate cancer. N Engl J Med. 2003;349:215-24.

4. Kulkarni GS, Al-Azab R, Lockwood G, Toi A, Evans A, Trachtenberg J, et al.: Evidence for a biopsy derived grade artifact among larger prostate glands. J Urol. 2006;175:505-9.

5. Liu JJ, Brooks JD, Ferrari M, Nolley R, Presti JC Jr: Small prostate size and high grade disease--biology or artifact? J Urol. 2011;185:2108-11. 
6. Briganti A, Chun FK, Suardi N, Gallina A, Walz J, Graefen M, et al.: Prostate volume and adverse prostate cancer features: fact not artifact. Eur J Cancer. 2007;43:2669-77.

7. Newton MR, Phillips S, Chang SS, Clark PE, Cookson MS, Davis $\mathrm{R}$, et al.: Smaller prostate size predicts high grade prostate cancer at final pathology. J Urol. 2010;184:930-7.

8. Ngo TC, Conti SL, Shinghal R, Presti JC Jr: Prostate size does not predict high grade cancer. J Urol. 2012;187:477-80.

9. Epstein JI, Allsbrook WC Jr, Amin MB, Egevad LL; ISUP Grading Committee. The 2005 International Society of Urological Pathology (ISUP) Consensus Conference on Gleason Grading of Prostatic Carcinoma. Am J Surg Pathol. 2005;29:1228-42.

10. Freedland SJ, Isaacs WB, Platz EA, Terris MK, Aronson WJ, Amling CL, et al.: Prostate size and risk of high-grade, advanced prostate cancer and biochemical progression after radical prostatectomy: a search database study. J Clin Oncol. 2005;23:7546-54.

11. Kassouf W, Nakanishi H, Ochiai A, Babaian KN, Troncoso $P$, Babaian RJ: Effect of prostate volume on tumor grade in patients undergoing radical prostatectomy in the era of extended prostatic biopsies. J Urol. 2007;178:111-4.

12. Borden LS Jr, Wright JL, Kim J, Latchamsetty K, Porter CR: An abnormal digital rectal examination is an independent predictor of Gleason > or $=7$ prostate cancer in men undergoing initial prostate biopsy: a prospective study of 790 men. BJU Int. 2007;99:559-63.

13. Thompson IM, Ankerst DP, Chi C, Lucia MS, Goodman PJ, Crowley JJ, et al.: Operating characteristics of prostatespecific antigen in men with an initial PSA level of $3.0 \mathrm{ng} / \mathrm{ml}$ or lower. JAMA. 2005;294:66-70.
14. Optenberg SA, Clark JY, Brawer MK, Thompson IM, Stein $\mathrm{CR}$, Friedrichs P: Development of a decision-making tool to predict risk of prostate cancer: the Cancer of the Prostate Risk Index (CAPRI) test. Urology. 1997;50:665-72.

15. Elliott CS, Shinghal R, Presti JC Jr.: The influence of prostate volume on prostate-specific antigen performance: implications for the prostate cancer prevention trial outcomes. Clin Cancer Res. 2009;15:4694-9.

16. Roehrborn CG, Andriole GL, Wilson TH, Castro R, Rittmaster RS: Effect of dutasteride on prostate biopsy rates and the diagnosis of prostate cancer in men with lower urinary tract symptoms and enlarged prostates in the Combination of Avodart and Tamsulosin trial. Eur Urol. 2011;59:244-9.

17. Epstein JI, Walsh PC, Carter HB: Dedifferentiation of prostate cancer grade with time in men followed expectantly for stage T1c disease. J Urol. 2001;166:1688-91.
Correspondence address: Murat Ustuner, MD University of Kocaeli Department of Urology Campus of Umuttepe, 41380, Kocaeli, TURKEY Fax: + 90262 303-8003 E-mail: muratustuner@gmail.com 